\title{
Endovascular rescue for malpositioned frozen elephant trunk into the false lumen
}

\author{
Daichi Takagi ${ }^{1}$, Takuya Wada ${ }^{2}$, Wataru Igarashi $^{2}$, Takayuki Kadohama $^{1}$, kentaro kiryu ${ }^{2}$, \\ Takeshi Arai $^{1}$, and Hiroshi Yamamoto ${ }^{2}$ \\ ${ }^{1}$ Akita University \\ ${ }^{2}$ Akita University Graduate School of Medicine School of Medicine
}

June 12,2021

\begin{abstract}
We describe a case of frozen elephant trunk deployment unintentionally malpositioned into the false lumen. An 83-year-old man underwent total arch repair with a frozen elephant trunk for type A acute aortic dissection complicated by mesenteric malperfusion. However, intraoperative transesophageal echocardiography showed expansion of the false lumen in the descending aorta, suggesting a malpositioned frozen elephant trunk into the false lumen. Endovascular fenestration of the dissecting flap and subsequent endograft deployment from the inside of the malpositioned frozen elephant trunk graft to the true lumen of the descending aorta was successfully performed under intravascular ultrasound guidance.
\end{abstract}

\section{Endovascular rescue for malpositioned frozen elephant trunk into the false lumen}

Daichi Takagi, MD, PhD, Takuya Wada, MD, Wataru Igarashi, MD, Takayuki Kadohama, MD, PhD, Kentaro Kiryu, MD, Takeshi Arai, MD, Hiroshi Yamamoto, MD, PhD

Department of Cardiovascular Surgery, Akita University Graduate School of Medicine, Hondo 1-1-1, Akita, 010-8543, Japan

Running head: Malpositioned FET graft

\section{Correspondence}

Daichi Takagi, MD, PhD, Department of Cardiovascular surgery, Akita University Graduate School of Medicine, Hondo 1-1-1, Akita, 010-8543, Japan.

Tel: +81-18-884-6135

Fax: +81-18-836-2625

E-mail: daichi0129@gmail.com

Prior Presentation: None.

Funding: None.

Conflict of Interests: None.

Abstract

We describe a case of frozen elephant trunk deployment unintentionally malpositioned into the false lumen. An 83-year-old man underwent total arch repair with a frozen elephant trunk for type A acute aortic dissection complicated by mesenteric malperfusion. However, intraoperative transesophageal echocardiography 
showed expansion of the false lumen in the descending aorta, suggesting a malpositioned frozen elephant trunk into the false lumen. Endovascular fenestration of the dissecting flap and subsequent endograft deployment from the inside of the malpositioned frozen elephant trunk graft to the true lumen of the descending aorta was successfully performed under intravascular ultrasound guidance.

\section{Introduction}

Frozen elephant trunk (FET) deployment for type A acute aortic dissection (TAAD) has been widely accepted because it induces aortic remodeling in the downstream aorta. ${ }^{1}$ FET malposition into the false lumen (FL) has rarely been reported. However, urgent intervention is required to prevent ischemic organ damage because endograft malposition has dire consequences for visceral, renal, and lower extremity perfusion. ${ }^{2,3}$ In this report, we describe the clinical features of this complication and the endovascular technique used to divert back persistent FL perfusion to the true lumen (TL) of the downstream aorta. The patient provided consent for this report.

\section{Case Report}

An 83-year-old man presented with acute-onset abdominal pain. Computed tomography (CT) revealed TAAD with primary entry tear at the distal aortic arch and compression of the TL by the FL in the superior mesenteric artery (SMA) with dilated and fluid-filled small bowel loops (Figure 1). Thus, the diagnosis was TAAD, complicated by mesenteric malperfusion. We decided to reperfuse the visceral organs before central aortic repair.

An emergency surgery was performed under general anesthesia. Laparotomy was performed, and a pulseless SMA and small bowel ischemia were identified. Under fluoroscopic guidance, a 10-mm stent (SMART; Cordis Corp., Miami Lakes, FL, USA) was deployed at the ostium of the SMA in a retrograde fashion through the 6 Fr sheath inserted from the branch of the SMA, which was then connected to the femoral artery sheath, which served as an external shunt. Thereafter, total arch repair with FET, "Zone 0 arch repair strategy," was performed using a FET graft and a 4-branched graft (35 $\mathrm{mm} \times 150 \mathrm{~mm}$ J Graft FROZENIX and $28 \mathrm{~mm}$ J Graft SHIELD, respectively; Japan Lifeline Co. Ltd., Tokyo, Japan). A primary entry tear was identified in the distal aortic arch (Figure 2A). The FET graft was carefully inserted into the descending aorta under direct vision. After weaning from cardiopulmonary bypass (CPB), the SMA remained pulseless. In addition, transesophageal echocardiography (TEE) showed expansion of the FL in the descending aorta distal to the FET graft, suggesting malposition of the FET into the FL of the descending aorta (Figure 2B). Thereafter, malposition of the FET was confirmed using intravascular ultrasound (IVUS).

Percutaneous fenestration of the dissecting flap and endograft deployment from the inside of the malpositioned FET to the TL of the descending aorta were subsequently performed. Under IVUS and fluoroscopic guidance, an angled 5-Fr catheter (Impress Diagnostic Peripheral catheter; Merit Medical, South Jourdan, UT, USA) was rotated and its tip was positioned against the center of the dissecting flap. Then the dissecting flap, $4 \mathrm{~cm}$ distal to the distal end of the FET, was perforated using a 0.014-inch tapered tip microguidewire (Chevalier14 Tapered 30; FMD Co., Ltd, Tokyo, Japan) by a quick, short thrust. The outer catheter was advanced over the microguidewire from the TL to the FL of the descending aorta. Thereafter, a 0.035-inch extra-stiff guidewire (Lunderquist; Cook Medical Inc., Bloomington, IN, USA) was reintroduced through the outer catheter, and a stiff guidewire was placed across the dissecting flap to the inside of the FET (Figure 2D/E/F). A tapered 38/34 mm $\times 190 \mathrm{~mm}$ endograft (Relay Plus; Bolton Medical, Sunrise, FL, USA) was deployed from the inside of the FET to the TL of the descending aorta. Subsequent aortography showed no endoleak, and IVUS revealed good expansion of the endograft and the TL of the downstream aorta. SMA pulsation and bowel peristalsis returned after endograft deployment. Postoperative complications related to aortic or visceral malperfusion were not observed. Follow-up CT revealed a patent endograft and expansion of the TL of the downstream aorta without SMA stenosis (Figure 3).

\section{Discussion}

Although extremely rare, FET malposition into the FL is reported to be a fatal complication. Most cases of 
FET malposition into the FL were diagnosed using postoperative CT. ${ }^{2-4}$ Tamai et al. reported a case of FET malposition diagnosed by TEE during circulatory arrest that was successfully treated by additional FET deployment into the TL. ${ }^{5}$ In our case, FET malposition was suspected after weaning from CPB by TEE and pulseless SMA. Although FET removal and re-deployment or additional FET deployment into the TL could be alternative options, we performed endovascular rescue because additional cardiac and circulatory arrest could cause excessive invasion.

Fenestration of the dissecting flap using a radio frequency system and subsequent endograft deployment has been reported as an effective technique in cases of chronic dissection. ${ }^{4}$ However, percutaneous endovascular fenestration and endografting for FET malposition in cases of acute dissection have never been reported. Because the dissecting flap architecture is much thinner in acute dissection than in chronic dissection, ${ }^{6}$ the dissecting flap can be easily perforated only by a tapered tip microguidewire through an angled catheter under IVUS and fluoroscopic guidance.

In conclusion, percutaneous endovascular fenestration of the dissecting flap and subsequent endograft deployment from the FET to the TL of the descending aorta under the guidance of IVUS are effective and less-invasive surgical treatments after FET malposition into the FL for TAAD.

\section{Author contributions}

Daichi Takagi wrote the manuscript; other authors surpervised the case.

The authors have obtained the patients' informed consent for publication. Institutional Review Board review is not applicable to this report.

\section{References}

1. Yamamoto H, Kadohama T, Yamaura G, et al. Total arch repair with frozen elephant trunk using the "zone 0 arch repair" strategy for type A acute aortic dissection. J Thorac Cardiovasc Surg . 2019;159:36-45. DOI:10.1016/j.jtcvs.2019.01.125.

2. Han SM, Gasper WJ, Chuter TAM. Endovascular rescue after inadvertent false lumen stent graft implantation. J Vasc Surg . 2016;63:518-522. DOI:10.1016/j.jvs.2014.11.072.

3. Fujii M, Watanabe H, Otsu M, Sugahara Y. Incorrect frozen elephant trunk deployment into the false lumen of a patient with complicated type B acute dissection. Eur J Cardiothorac Surg . 2019;55:1222-1224. DOI:10.1093/ejcts/ezy35\%.

4. Wong RHL, Yu PSY, Kwok MWT, et al. Endovascular fenestration for distal aortic sealing after frozen elephant trunk with thoraflex.Ann Thorac Surg . 2017;103:e479-e482. DOI:10.1016/j.athoracsur.2016.12.039.

5. Tamai K, Hori D, Yuri K, Yamaguchi A. Additional frozen elephant trunk as a bailout for a misdeployed frozen elephant trunk in the false lumen in a patient with acute aortic dissection. Eur $J$ Cardiothorac Surg. 2020;57:399-401. DOI:10.1093/ejcts/ezz213.

6. Peterss S, Mansour AM, Ross JA, et al. Changing pathology of the thoracic aorta from acute to chronic dissection: Literature review and insights. $J$ Am Coll Cardiol . 2016;68:1054-1065. DOI:10.1016/j.jacc.2016.05.091.

\section{Figure Legends}

Figure 1. Preoperative computed tomography (CT) revealed type A acute aortic dissection. (A) Threedimensional CT. (B) Primary entry tear located in the distal aortic arch. (C/D) True lumen of the ascending aorta, abdominal aorta, and superior mesenteric artery were severely compressed by the false lumen. Dilated and fluid-filled small bowel loops suggested mesenteric malperfusion.

Figure 2. (A) Primary entry tear was identified at the distal aortic arch under direct vision. (B) Intraoperative transesophageal echocardiography revealed true lumen (TL) stenosis and false lumen (FL) expansion in the downstream aorta. (C) Aortography. White and red arrows showed the levels of panels D, E, and F. (D, 
E, F) Intravascular ultrasound revealed FL expansion and TL stenosis at all levels. The stiff guidewire was introduced in the FL at the level just distal to the misdeployed frozen elephant trunk (D) across the dissecting flap at the perforation point (E; red arrow) and in the TL of the descending aorta (F). TL, true lumen; FL, false lumen

Figure 3. Postoperative computed tomography (CT). (A) An endograft was deployed from the inside of the FET to the TL of the descending aorta across the perforated dissecting flap (white arrow). (B, C, D) The FET, additional endograft, the true lumen of the SMA, and the abdominal aorta were patent without any stenosis

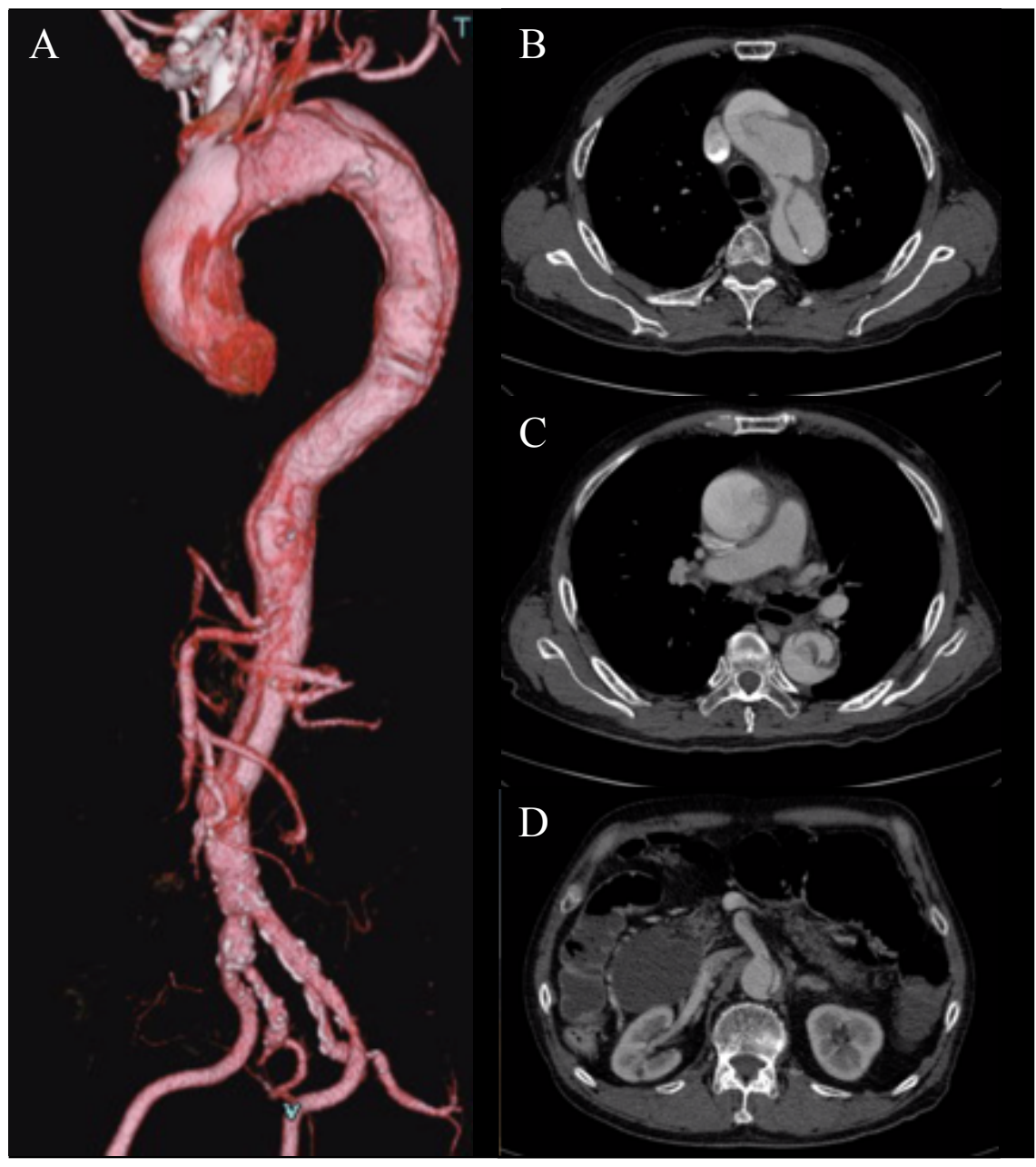




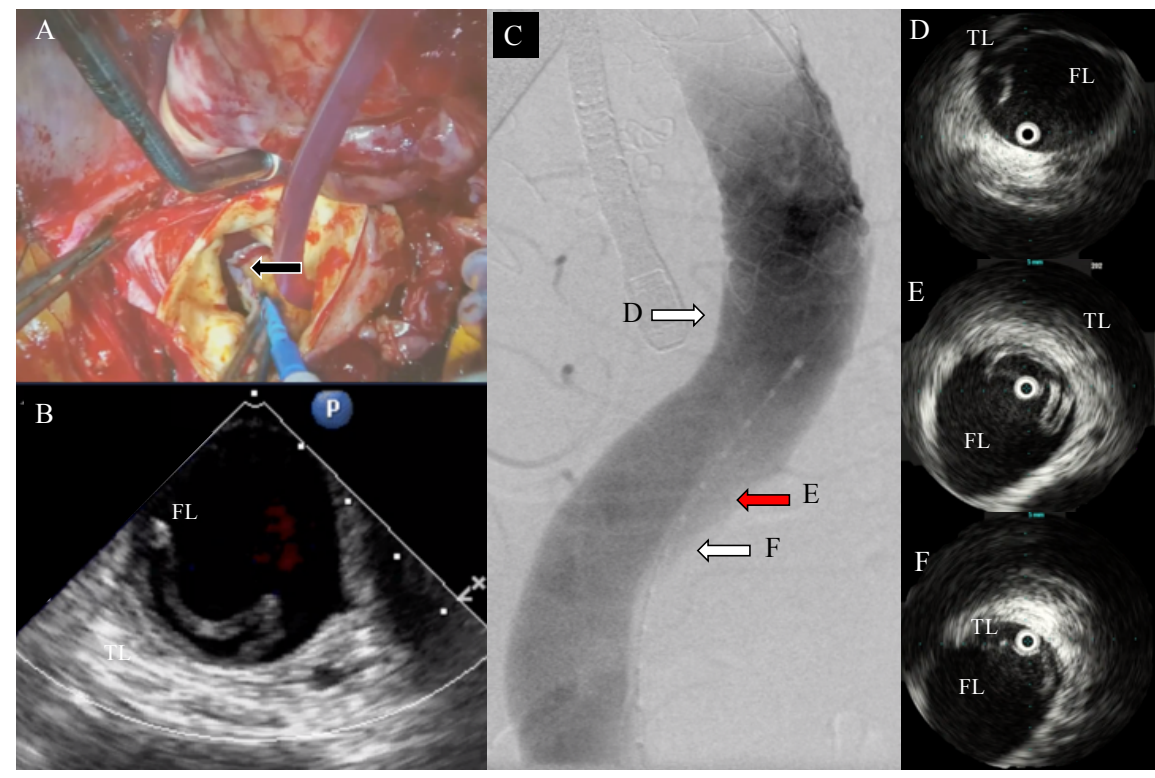




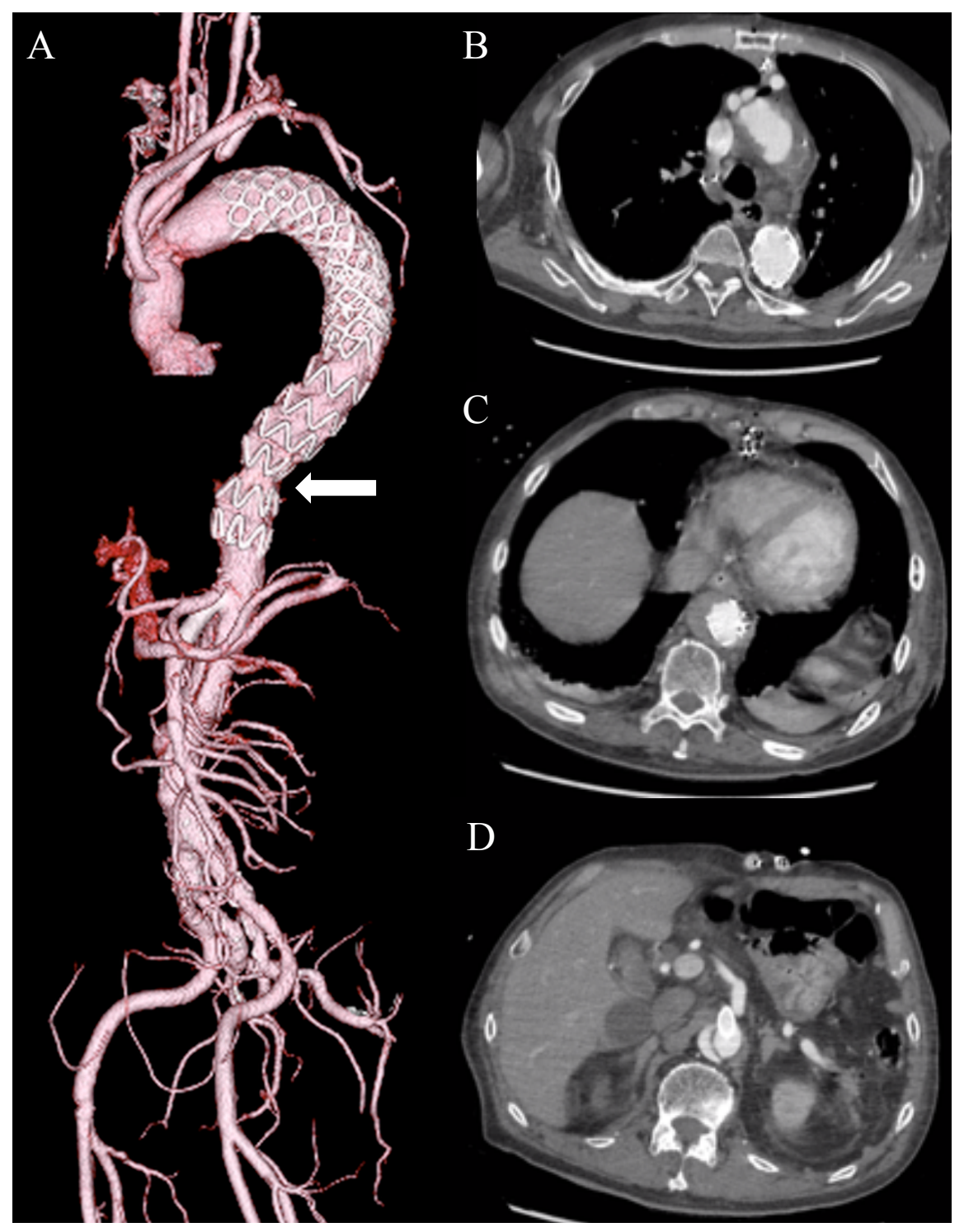

